\title{
Poliamore: una nuova parola per un vecchio concetto?
}

\section{Sara Giovine}

PUBBLICATO: 31 MARZO 2019

La parola poliamore indica la pratica (o la possibilità) di intrattenere più di una relazione intima (sessuale e/o sentimentale) contemporaneamente, con il consenso esplicito e consapevole di tutte le persone coinvolte. Dal punto di vista linguistico, si tratta di una voce composta dal confisso poli- (dal greco polýs 'molto'), usato spesso come primo elemento dei composti e indicante molteplicità numerica o quantitativa, e dal sostantivo amore, sul modello dellinglese polyamory (a sua volta formato dal prefisso di origine greca poly-e dal sostantivo latino amor 'amore' con l'aggiunta del suffisso nominale $-y$, sul modello di polygamy).

La nostra parola rappresenta infatti un calco del termine inglese polyamory, che viene coniato e usato per la prima volta negli Stati Uniti: come riportato in diversi saggi dedicati all'argomento, nel I99o Morning Glory Zell-Ravenheart (pseudonimo di Diana Moore), leader di una comunità neopagana americana, pubblica nella rivista "Green Egg Magazine", fondata col marito Oberon Zell-Ravenheart, un articolo intitolato A Bouquet of Lovers: strategies for responsible open relationships, in cui spiega i meccanismi del suo matrimonio aperto ed espone il suo ideale di relazione 'multipla', basata sul coinvolgimento sessuale e sentimentale di più persone contemporaneamente. Per definire tale tipo di relazione, da lei polemicamente contrapposto al modello sociale e sessuale dominante della monogamia, la Ravenheart, pur non ricorrendo ancora al sostantivo polyamory, si serve dell'aggettivo polyamorous, che forma unendo il prefisso poly- 'molti' e l'aggettivo amorous 'amoroso':

Honesty and willing Polyamorous Committment are the basic building blocks all partners must use to build a lasting Open Relationship. [...] Territorial jealousy has no place in a polyamorous agreement ['Lonestà e il volontario impegno poliamoroso sono gli elementi costitutivi di base a cui tutti i partner devono ricorrere per costruire una relazione aperta duratura. [...] Non c'è posto per la gelosia territoriale in un accordo poliamoroso'] (Morning Glory Zell, A Bouquet of Lovers, "Green Egg Magazine", XXIII, 89, May 1990).

La prima attestazione del sostantivo polyamory è di poco successiva e risale più precisamente al maggio del ' 92 , quando Jennifer L. Wesp crea sulla rete Usenet un newsgroup, ossia uno spazio di discussione virtuale, dedicato allo scambio di informazioni tra gli utenti interessati al tema delle relazioni amorose plurime, denominandolo proprio polyamory (alt.polyamory.org):

I propose to form the group alt.poly-amory. It would be a place for people who have multiple lovers to talk about the various problems unique to us ['Propongo di formare il gruppo di discussione alt.poly-amory. Sarà uno spazio per le persone che hanno amori plurimi per parlare dei diversi problemi che ci riguardano'] (J. L. Wesp, Proposal for alt.poly-amory, alt.config, Usenet newsgroup, 2I May 1992).

Il fenomeno esisteva naturalmente anche prima della coniazione della parola: secondo gli stessi fautori del poliamore, la sua origine andrebbe ricondotta al pensiero filosofico di Charles Fourier, pensatore francese vissuto tra la fine del Settecento e l'inizio dell'Ottocento, che nella sua opera Il nuovo mondo amoroso descrive, nell'ambito della società utopistica di Armonia, il superamento del modello familiare monogamico a favore di rapporti amorosi plurimi. Lopera, pubblicata postuma nel 1967, influenza fortemente il dibattito del tempo sui temi dell'amore libero e della liberazione dei costumi sessuali, specialmente negli ambienti hippie americani, in cui si hanno le prime sperimentazioni di vita comunitaria caratterizzate dall'instaurazione di relazioni aperte tra più partner. Prima degli anni 'go, forse anche per le dimensioni più circoscritte del fenomeno (socialmente meno accettato rispetto a oggi e generalmente associato a stili di vita alternativi, come appunto quello hippie), non si sentiva tuttavia l'esigenza di ricorrere a una parola specifica per denominarlo, per cui si parlava genericamente di relazioni aperte, o di relazioni non monogame, o ancora di non monogamia responsabile. Le cose cambiano con l'avvento di internet e la nascita dei primi siti e blog dedicati al tema, che contribuiscono alla crescente diffusione del fenomeno e alla formazione in rete di una vera e propria comunita di sostenitori delle relazioni 'poliamorose' (ed è infatti proprio in rete che, come abbiamo detto, viene usata per la prima volta la parola polyamory). Il poliamore è poi al centro del fortunatissimo libro delle psicologhe americane Dossie Easton e Janet Hardy, The Ethical Slut. A guide to Infinite Sexual Possibilities, pubblicato negli Stati Uniti nel I997 e poi riedito con 
ampi aggiornamenti nel 2009, una sorta di guida alle relazioni 'poliamorose' e a ogni altra forma di rapporto aperto, il cui successo concorre a rendere nota la filosofia del poliamore a un più vasto pubblico, oltre a contribuire a una più ampia circolazione del nuovo termine, che nel 2006 viene incluso (insieme alle forme corradicali polyamorous 'poliamoroso' e polyamorist 'fautore del poliamore') nell'Oxford English Dictionary.

In italiano la prima attestazione della parola poliamore risale al I998, quando viene impiegata in un'intervista del settimanale "L'Espresso" a una donna 'poliamorosa' di nome Jane, la cui storia era stata già resa nota da un saggio del sociologo Kevin Lano sui rapporti non monogamici, intitolato Breaking The Barriers To Desire: New Approaches To Multiple Relationships e pubblicato nel Regno Unito nel 1995. Trattandosi di un'intervista a una donna inglese, la prima apparizione della voce italiana rappresenta di conseguenza verosimilmente la semplice traduzione della parola originale effettivamente usata nel dialogo con l'intervistata:

In che cosa pensa che il poliamore sia diverso dalle comuni o dall'amore libero degli anni Sessanta? «Allepoca ero una bambina. Ma mi sembra che allora ci fosse un malinteso sulla concezione di amore libero inteso come promiscuità e sesso casuale. Ė per questo che non ha funzionato. Per noi si tratta di un profondo e serio rapporto d'amore con un mutuo accordo pensato e alimentato ogni giorno. C’e una consapevolezza diversa» (Annalisa Piras, In tre ci si ama meglio. Storia di Jane, che vive con due uomini, "L'Espresso", 44, p. 120, 3/10/1998).

Tale attestazione resta a lungo isolata, con l'eccezione di un paio di occorrenze sporadiche della fine del primo decennio del Duemila, riscontrate in articoli di quotidiani che danno notizia delle ultime pubblicazioni del sociologo ed economista francese Jacques Attali, teorico, oltre che convinto sostenitore del poliamore. Si tratta quindi anche in questi casi di primi tentativi di trasposizione in italiano della parola inglese, che si è nel frattempo diffusa anche in francese nella forma polyamour, usata da Attali nei suoi testi:

Per l'ottimo Jacques, il sentimento si apre oggi a più relazioni: "multiple e simultanee", precisa. Riverniciando con Internet l'antico piglio radicale, il vecchio economista socialista sostiene che l'amore dovrebbe addirittura cambiar nome e chiamarsi netloving, per analogia con il networking: dunque, poliamore, polifamiglia, polifedeltà (Alberta Marzotto, Netloving: l'amore ai tempi di Internet, Repubblica.it, I8/2/2008).

Bisogna infatti attendere il successivo decennio per una maggiore diffusione della parola, favorita dalla nascita, anche nel nostro paese, dei primi siti e blog dedicati al tema del poliamore: nel 2012 viene aperto il portale poliamore.org, che si propone come il principale punto di riferimento per gli interessati al tema delle relazioni amorose plurime in Italia, e nel 2012 il sito rifacciamolamore.it, che ha però una prospettiva più ampia, che interessa non solo il poliamore, ma anche ogni altra forma di relazione e di sessualità non convenzionale. Nel 2014 viene poi pubblicata la traduzione italiana del saggio della Hardy e della Easton, che riporta già nel titolo il riferimento al poliamore, che era invece assente in quello originale (La zoccola etica. Guida al poliamore, alle relazioni aperte e altre avventure), mentre negli anni successivi l'uscita di film, serie tv e reality che si occupano di relazioni 'poliamorose' (come il film-documentario Lutine del 20I6, il reality americano Polyamory, trasmesso in Italia dal canale Cielo sempre nel 2016, o la serie Netflix You Me Her del 2017) contribuisce a dare maggiore visibilità mediatica al fenomeno e comporta di conseguenza anche un deciso incremento dell'uso della nuova parola. Questa compare soprattutto all'interno di blog, riviste femminili e altre pubblicazioni online, che si propongono di spiegare in che cosa consista esattamente il fenomeno e come si differenzi da una più 'tradizionale' relazione aperta, mentre prevedibilmente più scarse risultano le attestazioni della parola nei quotidiani (2o le occorrenze riscontrate negli archivi della "Repubblica" e appena una decina quelle nel "Corriere della Sera" e nella "Stampa"), che si limitano in genere a dare notizia delle più recenti pubblicazioni sul tema. Per esempio:

Chiodo scaccia chiodo. L'ha presa alla lettera Emily Witt, giornalista statunitense, che a zo anni si è ritrovata single dopo una lunga relazione. E si è lanciata in una sperimentazione di tutte le nuove forme di amore e sesso, dalla meditazione orgasmica al poliamore. Risultato: il libro Future Sex (Minimum Fax, 2017) (Laura Aguzzi, Cuore spezzato? Sassolini e Gloria Gaynor, "la Stampa", sez. Società, 7/2/2018).

Nonostante la sua settorialità, il termine pare comunque essersi relativamente affermato nell'uso, quantomeno in quello del web: una ricerca condotta nelle pagine italiane di Google il 20/2/2019 ci restituisce infatti I42.00o risultati della parola, che è stata inoltre registrata da Treccani Neologismi 2018 e accolta nell'edizione 2018 del dizionario Garzanti. Alla fortuna della voce può aver contribuito l'analogia con i numerosi altri composti con poli- attestati nella nostra lingua, come politeismo, policlinico, poliestere, ma soprattutto con quelli relativi alla stessa sfera semantica di poliamore, come poligamia, che indica l'unione matrimoniale plurima di un uomo con più donne 
o di una donna con più uomini, per cui si ricorre anche, con dei termini più specifici, rispettivamente a poliginia (composto appunto da poli- e del greco gyné 'donna') e poliandria (dal greco polýandros 'che ha molti sposi', a sua volta da anér andrós 'uomo').

Va infine segnalato come, accanto a poliamore, risultino attestate nell'uso anche le varianti minoritarie poliamoria, che in alcuni portali e blog dedicati al tema viene ritenuta forma più vicina all'inglese polyamory e dunque preferibile rispetto alla più diffusa poliamore, epoliamorismo, che sarebbe però secondo alcuni non semplice sinonimo di poliamore, bensi termine indicante più precisamente la posizione filosofica di chi ammette la possibilità che una persona abbia più di un rapporto intimo. Abbastanza diffuse anche le forme derivate poliamoroso e poliamorista, usate in riferimento a chi pratica o sostiene la possibilità di intrecciare delle relazioni non monogame, mentre decisamente piu sporadiche si rivelano le attestazioni di altre voci composte con poli- a indicare persone o attività che abbiano una qualche attinenza con la pratica del poliamore: tali forme si configurano infatti, almeno per il momento, come formazioni effimere ed estemporanee, di uso circoscritto a quelle sezioni di blog e riviste femminili che si occupino del fenomeno. È il caso per esempio di poliaperitivo 'aperitivo tra persone che praticano o che sono interessate al poliamore'; polimeriggio 'incontro pomeridiano per discutere di poliamore e/o conoscere altre persone poliamorose'; policurioso 'persona interessata al poliamore, che però ancora non lo pratica'; e polifedele 'persona impegnata in una relazione chiusa con più di un/una partner'.

\section{Cita come:}

Sara Giovine, Poliamore: una nuova parola per un vecchio concetto?, "Italiano digitale", 2019, VIII, 2019/1 (gennaio-marzo), pp. 62-64.

DOI: $10.35948 / 2532-9006 / 2019.3086$

\section{Copyright 2019 Accademia della Crusca}

Pubblicato con licenza creative commons CC BY-NC-ND 